\title{
Mapping Wayfinding Behaviour in a Topographic Immersive Virtual Environment (TopoIVE)
}

\author{
Łukasz Halik ${ }^{\text {a }}$, Alexander J. Kent ${ }^{\text {b,* }}$ \\ ${ }^{a}$ Adam Mickiewicz University, Poznan, Poland lukasz.halik@amu.edu.pl \\ ${ }^{b}$ Canterbury Christ Church University, UK alexander.kent@ canterbury.ac.uk \\ * Corresponding author
}

Keywords: Virtual Reality Immersive Virtual Environment (IVE), 2D versus 3D, topographic data, wayfinding

\begin{abstract}
:
We investigate users' wayfinding behaviour in a Topographic Immersive Virtual Environment (TopoIVE) created from urban topographic data. The experiment involved 60 participants: 30 were given a navigational task and 30 were allowed to freely explore a simulated city. Participants used a Head-Mounted Display (HMD) Virtual Reality (VR) app to enable them to switch between 2D and 3D modes of visualization. Their locations within the TopoIVE were recorded and analysed.
\end{abstract}

Although several participant variables were tested (including their VR experience), the results reveal that different wayfinding solutions were adopted by males and females. The findings also suggest that users referred to both $2 \mathrm{D}$ and 3D modes of visualization in their wayfinding strategy. We therefore recommend that developers of virtual environments should consider how different strategies are adopted by different users.
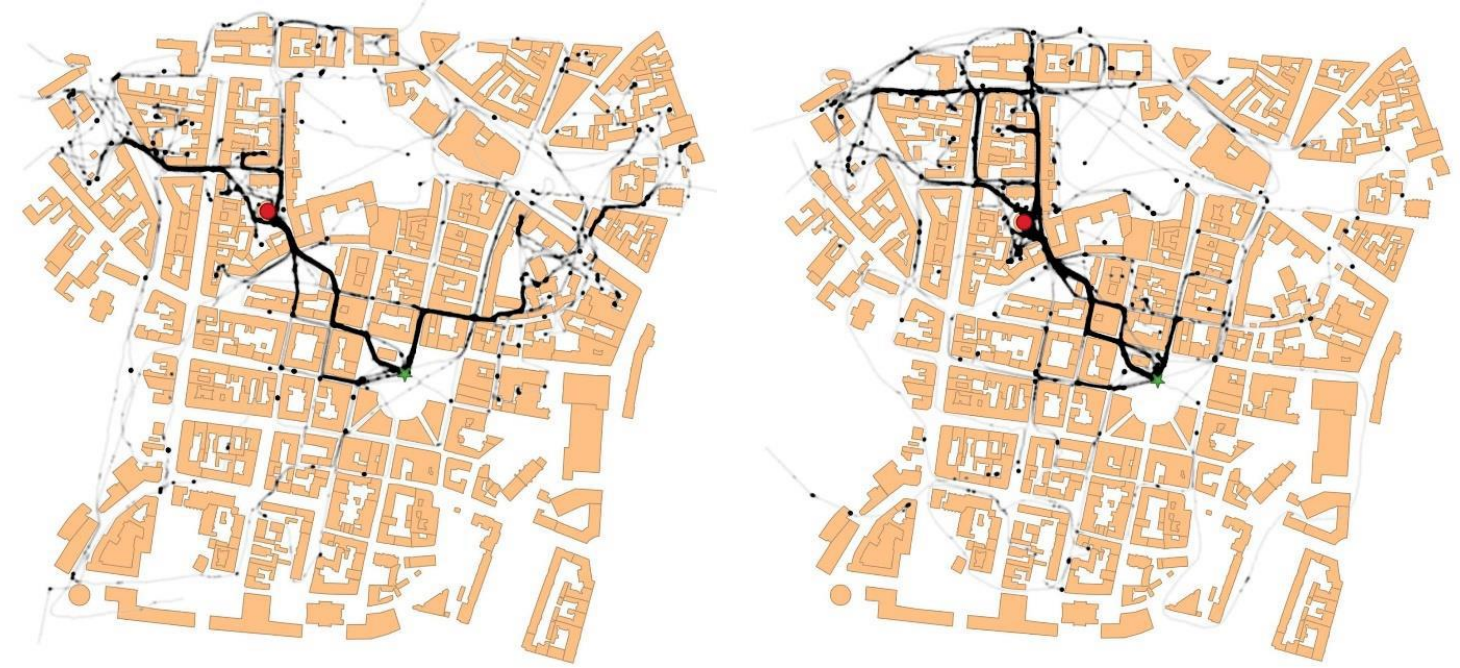

Figure 1. Wayfinding tracks of male (left) and female (right) participants in the study. 